\title{
Evolution of the Prevalence, Clinical Features and Risk Factors for Acute Mountain Sickness in the Qinghai-Tibet Plateau
}

\section{Chen Zhang}

Third Military Medical University Second Affiliated Hospital: Xinqiao Hospital

\section{Chuan Liu}

Third Military Medical University Second Affiliated Hospital: Xinqiao Hospital

\section{Renzheng Chen}

Third Military Medical University Second Affiliated Hospital: Xinqiao Hospital

Jie Yang

Third Military Medical University Second Affiliated Hospital: Xinqiao Hospital

\section{Jingbin Ke}

Third Military Medical University Second Affiliated Hospital: Xinqiao Hospital

\section{Shizhu Bian}

Third Military Medical University Second Affiliated Hospital: Xinqiao Hospital

\section{Shiyong Yu}

Third Military Medical University Second Affiliated Hospital: Xinqiao Hospital

\section{Jihang Zhang}

Third Military Medical University Second Affiliated Hospital: Xinqiao Hospital Jie Yu

Third Military Medical University Second Affiliated Hospital: Xinqiao Hospital

\section{Ying Zeng}

Third Military Medical University Second Affiliated Hospital: Xinqiao Hospital

\section{Xubin Gao}

Third Military Medical University Second Affiliated Hospital: Xinqiao Hospital

\section{Xiaohan Ding}

Third Military Medical University Second Affiliated Hospital: Xinqiao Hospital

\section{Yuanqi Yang}

Third Military Medical University Second Affiliated Hospital: Xinqiao Hospital

\section{Fangzhengyuan Yuan}

Third Military Medical University Second Affiliated Hospital: Xinqiao Hospital

\section{Chunyan He}

Third Military Medical University Second Affiliated Hospital: Xinqiao Hospital

\section{Yang Shen}


Third Military Medical University Second Affiliated Hospital: Xinqiao Hospital

\section{Hu Tan}

Third Military Medical University Second Affiliated Hospital: Xinqiao Hospital

\section{Xiaohui Zhao}

Third Military Medical University Second Affiliated Hospital: Xinqiao Hospital

\section{Jun Jin}

Third Military Medical University Second Affiliated Hospital: Xinqiao Hospital

\section{Lan Huang ( $\sim$ huanglan260@126.com )}

The Second Affiliated Hospital, Third Medical University (Army Medical University) https://orcid.org/0000-0001-6200-2309

\section{Research}

Keywords: high altitude, acute mountain sickness, risk factors, ascent protocol

Posted Date: July 13th, 2021

DOl: https://doi.org/10.21203/rs.3.rs-663450/v1

License: (1) (1) This work is licensed under a Creative Commons Attribution 4.0 International License. Read Full License 


\section{Abstract}

Background Ascending to high altitude (HA) contribute to the development of acute mountain sickness (AMS). The prevalence, clinical features and relative risks for AMS may dynamically evolve but has not been described.

Methods A total of 1,629 healthy men aged from 16 to 45 years were enrolled. A fast ascent cohort $(n=1283$ ) ascended to $3700 \mathrm{~m}$ by airplane and further ascended to $4400 \mathrm{~m}$ by bus; a slow ascent cohort ( $n=346$ ) ascended to $3450 \mathrm{~m}$ by bus and further ascended to $4100 \mathrm{~m}$ by bus. AMS was diagnosed by the Lake Louise Scoring (LLS) system.

Results As diagnosed by the old LLS and new LLS, the prevalence of AMS was $68.4 \%$ and $56.1 \%$ at 3700 $\mathrm{m}$, respectively, which decreased to $21.4 \%$ and $14.3 \%$ after further ascending to $4400 \mathrm{~m}$ in the fast ascent cohort; the prevalence of AMS was $30.3 \%$ and $25.7 \%$ at $3450 \mathrm{~m}$, which increased to $36.6 \%$ and $32.2 \%$ after further ascending to $4100 \mathrm{~m}$ in the slow ascent cohort. Moreover, AMS-related symptoms, such as headache, dizziness and fatigue, were the leading symptoms except at $4400 \mathrm{~m}$ in the fast ascent cohort. Fast ascent protocol was a risk factor during the initial ascent but became a protective factor after a further ascent to a higher altitude.

Conclusions Ascent protocol was the primary factor that affected the incidence of AMS and related symptoms. These findings imply a novel strategy for high altitude travels or work with a plan to ascend to a higher altitude.

\section{Background}

The number of sea level residents engaged in leisure, work or sports at high altitude (HA) is dramatically increasing. However, within the first few days after arrival at HA above $2500 \mathrm{~m}, 40-90 \%$ of unacclimated individuals may suffer from acute mountain sickness (AMS), depending on the altitude reached, the ascending rate, personal susceptibility, and degree of acclimatization [1]. If AMS is not treated appropriately, it may progress to life-threatening high-altitude pulmonary oedema (HAPE) or high-altitude cerebral oedema (HACE) [2]. Annually, AMS threatens the well-being and/or life of millions of sojourners and workers at $\mathrm{HA}$, which subsequently hinders the development of social economy [3]. For these reasons, illuminating the prevalence, clinical features and relative risks for AMS is of great importance for the prediction, prevention and treatment of AMS.

The symptoms of AMS are usually non-specific but incapacitating and include headache, gastrointestinal (GI) upset, fatigue, dizziness, insomnia, and so on [4]. However, due to the poor association with the other symptoms of AMS, insomnia is no longer the defining symptom for AMS diagnosis by the Lake Louise Scoring (LLS) system, which was revised in 2018 [5]. Theoretically, compared with the old LLS, the prevalence of AMS diagnosed by the newly revised LLS will be less frequent. Nevertheless, the prevalence, clinical features and relative risks for AMS defined by the new LLS-AMS and the differences between the old and new versions are still unknown. 
Identifying the risk factors for AMS is vital and challenging because of miscellaneous contributing factors. A previous study showed that the critical factors that influenced the risk of developing AMS were the altitude attained and the ascent rate. The prevalence of AMS increased with higher altitudes, and the risk of developing AMS was higher for fast ascents (faster than $400 \mathrm{~m}$ per day) than for slow ascents [6]. In addition, some demographic characteristics, such as age, sex, nationality, body mass index (BMI), and history of smoking have been evaluated and demonstrated to contribute to AMS. Additionally, some previous research revealed that the development of AMS declined when there were pre-acclimatization or prolonged acclimatization schedules [7]. Moreover, there is not always only a target altitude for individuals ascending to HA. Commonly, they may ascend to a higher altitude for various purposes after a short-term stay at an intermediate altitude. Therefore, the prevalence, clinical features and relative risks for AMS may dynamically evolve. However, this dynamic evolution has not been described to date.

For these reasons, the present study aimed to reveal the dynamics of the prevalence, clinical features and relative risks for AMS based on the old LLS and new LLS in a large population visiting above $3000 \mathrm{~m}$ with a fast or slow ascent protocol and further ascending to above $4000 \mathrm{~m}$.

\section{Methods}

\section{Study design and participants}

The present study included two prospective cohorts at the Qinghai-Tibet Plateau in June-July 2012 and June-July 2013. A total 1629 healthy men who were born or permanently lived in the lowland $(<500 \mathrm{~m}$, above sea level, asl) were recruited in June $2012(n=1283)$ and June $2013(n=346)$. For all subjects enrolled, a comprehensive medical examination was performed before the expedition. Subjects with any clinical conditions that may exhibit HA-related symptoms were excluded, including known pulmonary diseases, cardiovascular diseases, any chronic cardiovascular therapy, haematologic diseases, liver and kidney dysfunction, malignant tumours, and so on. Subjects with psychiatric disorders that prevented the completion of the data collection and subjects with high-altitude cerebral oedema or high-altitude pulmonary oedema needing emergency treatment were also excluded.

\section{Ascending procedures}

For the 2012 cohort, named the "fast ascent cohort", 1283 subjects successively ascended to HA from $500 \mathrm{~m}$ (Chengdu, Sichuan, China) to $3700 \mathrm{~m}$ (Lhasa, Tibet, China) by airplane in approximately $2 \mathrm{~h}$. After staying for 7 days at $3700 \mathrm{~m}$, they further ascended to $4400 \mathrm{~m}$ (Yangbanjin, Tibet, China) by bus. Among them, 387 subjects who did not further ascend to $4400 \mathrm{~m}$ and 138 subjects with incomplete questionnaires were excluded. Finally, the data from 757 subjects were collected after further ascending to $4400 \mathrm{~m}$.

For the 2013 cohort, named the "slow ascent cohort", 346 subjects successively ascended to $3450 \mathrm{~m}$ (Xinduqiao, Sichuan, China) from $400 \mathrm{~m}$ (Yanggongqiao, Chongqing, China) by bus within 4 days in a staged ascent mode. After staying for 3 days at $3450 \mathrm{~m}$, they further ascended to $4100 \mathrm{~m}$ (Litang, 
Sichuan, China) by bus. Among them, 21 subjects who refused to ascend further to $4100 \mathrm{~m}$ and 11 subjects with incomplete questionnaires were excluded. Finally, the data from 314 subjects were collected after the further ascent to $4100 \mathrm{~m}$ (Figure 1).

\section{Data collection}

For the 2012 cohort, data collection was performed on 15 June 2012 at SL and 18-24 h after arriving at $3700 \mathrm{~m}$ or $4400 \mathrm{~m}$. For the 2013 cohort, data collection was performed on $19^{\text {th }}$ June 2013 at SL and 18$24 \mathrm{~h}$ after arriving at $3450 \mathrm{~m}$ or $4100 \mathrm{~m}$. Structured case report form questionnaires were used to record demographic information, including age, height, weight, smoking, nationality, educational level and history of HA exposure within 1 year. The BMI was calculated as weight $(\mathrm{kg}) /[\text { height }(\mathrm{m})]^{2}$. Clinical symptoms were self-reported, including headache, dizziness, gastrointestinal upset, insomnia, fatigue, paraesthesia, constipation, dyspnoea, cough, chest distress, palpitation, tinnitus, dazzling, lethargy, and reduced activity.

\section{Diagnosis of AMS}

The incidence of AMS was identified by using the standard LLS as previously validated [8]. The severity of the AMS symptoms was scored from 0 (no discomfort) to 3 (severe discomfort) according to the participants' self-report. Participants were requested to complete the self-reported LLS before sleeping and after awakening, and the highest scores during the stay at HA were considered as the final LLS. In the present study, two criteria were used to define AMS: old LLS-AMS and new LLS-AMS. The latter was based on the old LLS-AMS without scoring the symptom of sleep disorders according to the newly revised diagnostic criterion [9]. Accordingly, subjects with LLS $\geq 3$ in the presence of headache were diagnosed with AMS.

\section{Statistical Analysis}

All the statistical analyses were conducted using SPSS 22.0 (IBM Corp., Armonk, NY, USA). Demographic characteristics, clinical symptoms and the incidence of AMS were presented as the mean \pm standard deviation or medians $\left(25^{\text {th }}-75^{\text {th }}\right.$ percentile) for continuous variables and compared using Student's $t$ tests or the Mann-Whitney $U$ test according to their normality following the Kolmogorov-Smirnov test. Categorical variables were expressed as counts and percentages and analysed using the $\chi^{2}$ test or Fisher's exact test, as appropriate. For the risk factor analysis, univariate and multivariate logistic regression analysis were performed. Demographic parameters were adjusted in the multivariate analysis. Moreover, to reduce the effect of potential confounding factors in the present study, propensity scorematching was performed for rigorous adjustment for significant differences in the baseline characteristics. Statistical power was calculated by PASS software (version 11, NCSS, LLC, Kaysville, UT, USA), and more than $80 \%$ statistical power was achieved between subgroups using a two-sided alpha of 0.05 . A two-sided $p$-value of $<0.05$ was considered statistically significant. 


\section{Ascending Procedures}

For the 2012 cohort, named the "fast ascent cohort", 1283 subjects successively ascended to HA from $500 \mathrm{~m}$ (Chengdu, Sichuan, China) to $3700 \mathrm{~m}$ (Lhasa, Tibet, China) by airplane in approximately $2 \mathrm{~h}$. After staying for 7 days at $3700 \mathrm{~m}$, they further ascended to $4400 \mathrm{~m}$ (Yangbanjin, Tibet, China) by bus. Among them, 387 subjects who did not further ascend to $4400 \mathrm{~m}$ and 138 subjects with incomplete questionnaires were excluded. Finally, the data from 757 subjects were collected after further ascending to $4400 \mathrm{~m}$.

For the 2013 cohort, named the "slow ascent cohort", 346 subjects successively ascended to $3450 \mathrm{~m}$ (Xinduqiao, Sichuan, China) from $400 \mathrm{~m}$ (Yanggongqiao, Chongqing, China) by bus within 4 days in a staged ascent mode. After staying for 3 days at $3450 \mathrm{~m}$, they further ascended to $4100 \mathrm{~m}$ (Litang, Sichuan, China) by bus. Among them, 21 subjects who refused to ascend further to $4100 \mathrm{~m}$ and 11 subjects with incomplete questionnaires were excluded. Finally, the data from 314 subjects were collected after the further ascent to $4100 \mathrm{~m}$ (Fig. 1).

\section{Results}

\section{Subject characteristics}

The baseline demographic characteristics are shown in Table 1. Subjects in the fast ascent cohort were slightly older than those in the slow ascent cohort $(23.5 \pm 4.2$ vs. $21.3 \pm 3.1$ years, $p<0.001)$, and the BMI of subjects in the fast ascent cohort was slightly higher than that in the slow ascent cohort $(21.8 \pm 2.2 \mathrm{vs}$. $21.2 \pm 1.9 \mathrm{~kg} / \mathrm{m}^{2}, \mathrm{p}<0.001$ ), which was mainly attributed to their differences in body weight. Moreover, there were fewer previous smokers and Han subjects in the fast ascent cohort than in the slow ascent cohort. Additionally, higher education levels were found, and more individuals had a history of HA exposure in the fast ascent cohort than in the slow ascent cohort (S1 Table).

\section{Incidence of clinical outcomes}

When diagnosing AMS by the old LLS, 877 subjects (68.4\%) in the fast ascent cohort developed AMS after the initial ascent to $3700 \mathrm{~m}$, which decreased to $21.4 \%$ after further ascent to $4400 \mathrm{~m}$, while 105 subjects (30.3\%) in the slow ascent cohort developed AMS after the initial ascent to $3450 \mathrm{~m}$, which increased to $36.6 \%$ after the further ascent to $4100 \mathrm{~m}$. However, when diagnosing AMS by the new LLS, 720 subjects $(56.1 \%)$ in the fast ascent cohort developed AMS after the initial ascent to $3700 \mathrm{~m}$, which decreased to $14.3 \%$ after the further ascent to $4400 \mathrm{~m}$, while 89 subjects $(25.7 \%)$ in the slow ascent cohort developed AMS after the initial ascent to $3450 \mathrm{~m}$, which increased to $32.2 \%$ after the further ascent to $4100 \mathrm{~m}$ (Table 1).

Among the 15 clinical symptoms that developed, headache (75.1\%) was most common in the fast ascent cohort after the initial ascent to $3700 \mathrm{~m}$, followed by dizziness, fatigue, activity reduction, and insomnia (Figure 2. A). However, after the further ascent to $4400 \mathrm{~m}$, activity reduction (42.4\%) became the leading 
symptom, followed by dizziness, fatigue, insomnia, dyspnoea and headache (Figure 2. B). Interestingly, dizziness (49.7\%) was most common in the slow ascent cohort after the initial ascent to $3450 \mathrm{~m}$, followed by headache, fatigue, activity reduction, and paraesthesia (Figure 2. C). However, the order of the first five clinical symptoms was not markedly changed after further ascent to $4100 \mathrm{~m}$ (Figure 2. D).

The results from the symptomatic analysis showed that the incidences of clinical symptoms including headache, dizziness, gastrointestinal upset, insomnia, fatigue, paraesthesia, dyspnoea, cough, chest distress, palpitation, dazzling, lethargy and activity reduction in subjects who quickly ascended to an intermediate altitude $(3700 \mathrm{~m})$ were higher than those who slowly ascended to an intermediate altitude $(3450 \mathrm{~m})$. However, after ascending to the higher altitude, the incidences of clinical symptoms, including headache, dizziness, fatigue, paraesthesia and tinnitus in the fast ascent cohort, were lower than those in the slow ascent cohort, except for insomnia. Additionally, in the fast ascent cohort, the incidences of almost all clinical symptoms were significantly decreased after further ascent to a higher altitude, except

for constipation and tinnitus. Nevertheless, in the slow ascent cohort, the incidences of fatigue, dyspnoea, cough and activity reduction were even increased after further ascent to a higher altitude (Table 1).

\section{Risk factors for the incidence of AMS}

The results from logistic regression suggested that age was only associated with the incidence of AMS at $4400 \mathrm{~m}$ in the fast ascent cohort when the old LLS was used for AMS diagnosis. Moreover, in the fast ascent cohort, current smoking was a protective factor (OR: $0.74,95 \% \mathrm{Cl}: 0.56$ to 0.97 ) at $3700 \mathrm{~m}$ in the fast ascent cohort when the old LLS was used but became a risk factor (OR: 1.95, 95\% Cl: 1.10 to 3.45) at $4100 \mathrm{~m}$ in the slow ascent cohort when the new LLS was used. Additionally, the Chinese non-Han population always acted as a protective factor at $4400 \mathrm{~m}$ or $3450 \mathrm{~m}$ when the old LLS was used or at $4100 \mathrm{~m}$ when the new LLS was used (Table 2a and Table 2b).

\section{The outcomes after propensity score-matching}

The propensity score-matched subjects among the overall population, fast and slow ascent cohorts showed no significant differences in the baseline demographic characteristics (S2 Table). There were no significant changes in the prevalence of AMS and its clinical features under different ascent protocols or diagnostic criteria, as well as their evolutions after further ascent to higher altitudes after propensity score-matching compared with that before propensity score-matching (S3 Table, Figure 2. $\left.A^{\prime}-D^{\prime}\right)$. However, after propensity score-matching, the ascent protocol was the most important factor affecting the incidences of AMS and its related symptoms (S4 Table). Irrespective of whether the old or new LLS was used for AMS diagnosis, the fast ascent protocol was a risk factor during the initial ascent but became a protective factor after further ascent to a higher altitude in the univariate and multivariate logistic regression models. Furthermore, these associations and risk evolutions have also been found for the incidences of headache, dizziness and fatigue, but not for the incidences of gastrointestinal upset and insomnia (Table 3). 


\section{Discussion}

The major findings of our present study were as follows: 1) In the fast ascent cohort, the prevalence of AMS was $68.4 \%$ and $56.1 \%$ at $3700 \mathrm{~m}$, which decreased to $21.4 \%$ and $14.3 \%$ when further ascending to $4400 \mathrm{~m}$, as diagnosed by the old LLS and new LLS, respectively; 2 ) In the slow ascent cohort, the prevalence of AMS was $30.3 \%$ and $25.7 \%$ at $3450 \mathrm{~m}$, which increased to $36.6 \%$ and $32.2 \%$ when further ascending to $4100 \mathrm{~m}$, as diagnosed by the old LLS and new LLS, respectively; 3) AMS-related symptoms such as headache, dizziness, and fatigue were the leading symptoms after the initial ascent to $3700 \mathrm{~m}$ or $3450 \mathrm{~m}$ and were still the main symptoms after further ascending to $4100 \mathrm{~m}$ in the slow ascent cohort but not to $4400 \mathrm{~m}$ in the fast ascent cohort; and 4) the ascent protocol was the primary factor that affected the incidence of AMS and related symptoms, showing that the slow ascent protocol was protective during the initial ascent but became a risk factor after further ascending to a higher altitude.

AMS is a common medical problem that affects the well-being of a large population. A recent systematic review summarized the published incidences of AMS from 53603 subjects, which demonstrated that the median AMS incidence was $60 \%$ in randomized trials, $51 \%$ in cohort studies, and $32 \%$ in cross-sectional studies. The results from the multivariate analysis suggested that the study design, mode of ascent, maximum altitude, population, and geographical location were significantly associated with the incidence of AMS [10]. Indeed, our present data on AMS prevalence were higher or lower than those of previous studies mainly in the population characteristics, mode of ascent, altitude reached and AMS definitions. Similar to our protocol, it had been reported that the incidence of AMS was $84 \%$ among tourists who ascended directly to $3740 \mathrm{~m}$ by airplane, whereas the incidence of AMS was $61 \%$ in subjects who ascended to the same altitude from $<3000 \mathrm{~m}$ by foot [11], which was consistent with our present results that subjects ascending to HA by plane reported more frequent AMS than with other protocols. Furthermore, previous results showed that the overall incidence of AMS was $53 \%$ after ascending to 4243 $\mathrm{m}$ altitude at Pheriche in the Himalayas of Nepal. It had also been reported that the prevalence of acute mountain sickness was $9 \%$ at $2850 \mathrm{~m}, 13 \%$ at $3050 \mathrm{~m}$, and $34 \%$ at $3650 \mathrm{~m}$ [12]; the latter of the three values was comparable with our prevalence in the slow ascent cohort at $3450 \mathrm{~m}$. Moreover, it had been demonstrated that the incidence of AMS was $59 \%$ of children trekking at $3952 \mathrm{~m}$ (Jade Mountain, Taiwan), which was consistent with our results that the most common AMS symptom was headache, followed by fatigue, sleep disorders, dizziness or lightheadedness and gastrointestinal upset [13].

After arriving at a new altitude above $2500 \mathrm{~m}$ in unacclimated individuals, AMS patients usually developed a group of nonspecific symptoms. Among these various symptoms, headache was the defining and indispensable symptom for AMS diagnosis [14]. After sequencing the 15 clinical symptoms reported in our present study, the main symptoms of AMS, including headache, dizziness and fatigue, remained as the cardinal symptoms after the initial ascent to $3700 \mathrm{~m}$ by plane or $3450 \mathrm{~m}$ by bus, which was consistent with previous results [15]. However, after further ascending to $4400 \mathrm{~m}$ in the fast ascent cohort, fatigue, headache and dizziness were no longer the leading symptoms, and their incidence was also lower than that in the slow ascent cohort after further ascending to $4100 \mathrm{~m}$, which suggested that the fast ascent protocol might be more effective in attenuating the incidence of AMS-related symptoms 
than the slow ascent protocol when subjects further ascended to a higher altitude after a short term of staying at the intermediate altitude. Additionally, activity reduction, which was not a symptom for AMS diagnosis, was another more frequent complaint. Consistently, a previous review claimed that individuals with AMS were frequently incapacitated [16]. Although insomnia had been excluded from the newly revised AMS diagnostic criteria, the incidence of insomnia was still prevalent, as previously described, and was more frequent in the fast ascent cohort than in the slow ascent cohort in our study.

Consistently, the incidence of AMS was highly correlated with the speed of ascent and age, but not sex or previous altitude experience [16]. A previous systematic review summarized that the fast ascent protocol had an OR of 4.69 (95\% Cl, 2.79-7.90), whereas a slow ascent protocol had an OR of $0.30(95 \% \mathrm{Cl}, 0.20-$ 0.44 ) for the incidence of AMS, which was highly consistent with our present results during the initial ascent to $3700 \mathrm{~m}$ or $3450 \mathrm{~m}$ by plane or by bus, respectively. In addition, compared with non-smokers, smokers showed a lower incidence of AMS, suggesting that smoking slightly lowers the risk of AMS but impairs long-term altitude acclimation during prolonged HA exposure [17], which was similar to our present findings that smoking was a protective factor for AMS in subjects who rapidly ascended to 3700 $\mathrm{m}$, but increased the risk for AMS in a prolonged HA exposure in the slow ascent cohort at $4100 \mathrm{~m}$. However, our data suggested that the associations of smoking and AMS were uncertain and depended on the ascent protocol, altitude attained and diagnostic criteria. Indeed, a previous meta-analysis also showed that there were no consistent results demonstrating that cigarette smoking acted as either a protective factor against or a risk factor for AMS [18].

It had been recommended that AMS could be prevented by ascending slowly at altitudes above $3000 \mathrm{~m}$ and taking a rest day every 3-4 days [19]. Such a protocol had been described as a "staged ascent", which had been considered the best method for AMS prevention [16]. Indeed, our present data also demonstrated that the incidence of AMS was definitely lower in the slow ascent cohort than in the fast ascent cohort after their initial ascent to an intermediate altitude. A previous report showed that 2 days of staging at an intermediate altitude lowered the incidence of AMS compared with the direct ascent group [20]. However, such approaches did not reduce the incidence of AMS with a rapid ascent to higher altitudes [21]. Therefore, the results from studies aiming to identify the benefit of intermittent hypoxic exposures for AMS prevention were somewhat conflicting for subjects planning a further ascent to higher altitudes. In our present study, the results from the field study at HA indicated that there was no significant impact of the slow ascent protocol combined with 3 days of staging at an intermediate altitude on the incidence of AMS after a further ascent to above $4000 \mathrm{~m}$. Interestingly, although the incidence of AMS was high in subjects after the fast ascent to the initial altitude by plane, the number of subjects suffering from AMS after a further ascent to a higher altitude was significantly lower than that of the initial ascent in the fast ascent cohort and that of a further ascent in the slow ascent cohort. These findings might imply a novel strategy for AMS prevention in individuals travelling to or working at HA when a further ascent to a higher altitude was needed.

\section{Limitations}


There were also several limitations. First, the enrolled participants were young, healthy men, and whether the established results can extend to other types of individuals or circumstances (such as women, older adults, children) is still unknown. Second, the effects of other modes of transport (such as the train) on the incidence of AMS under the present conditions are also unknown. Third, the classification of AMS was based on a self-report without an immediate medical control, which might lead to possible classification bias. Finally, due to the reality of the field study at HA, the target altitudes were not absolutely the same between the two cohorts. Theoretically, the incidence of AMS would be higher in subjects at higher target altitudes [22]; thus, our conclusions would be even more affirmative if the target altitudes of further ascent were the same in the two cohorts.

\section{Conclusions}

Our large cohort study showed the prevalence of AMS was $56.1 \%$ at $3700 \mathrm{~m}$, which decreased to $14.3 \%$ after further ascending to $4400 \mathrm{~m}$ in the fast ascent cohort; and the prevalence of AMS was $25.7 \%$ at $3450 \mathrm{~m}$, which increased to $32.2 \%$ after further ascending to $4100 \mathrm{~m}$ in the slow ascent cohort. The ascent protocol was the primary factor that affected the incidence of AMS and related symptoms, showing that a fast ascent protocol, which acted as a risk factor during the initial ascent, was converted into a protective factor after further ascending to a higher altitude. These findings imply a novel strategy for HA travels or work with a plan to further ascend to a higher altitude.

\section{Abbreviations}

HA: high altitude; AMS: acute mountain sickness; HAPE: high-altitude pulmonary oedema; HACE: highaltitude cerebral oedema; BMl: body mass index.

\section{Declarations}

\section{Ethics statements and registration}

This study was approved by the Clinical Research Ethics Board at the Army Medical University (Third Military Medical University No. 2012014) and was conducted in agreement with the Declaration of Helsinki. All subjects volunteered to participate in this study and gave written informed consent. This study was registered with the Chinese Clinical Trial Registration (No. ChiCTR-RCS-12002232, http://www.chictr.org.cn).

Consent for publication: Not applicable.

Availability of data and materials: The datasets generated for this study are available on request to the corresponding author.

Competing interests: None. 
Funding: This work is supported by grants from the National Natural Science Foundation of China (Grant No: 81730054), Military Logistics Research Project, PLA (Grant No: BLJ18J007) and the Special Health Research Project, Ministry of Health of PR China (Grant No: 201002012).

Authors' contributions: Prof. Lan Huang are guarantors and contributes to the study design. Dr. Chuan Liu and Chen Zhang analyze the data and draft the manuscript. All authors contribute to the interpretation of the results and approve the final version of the manuscript.

Acknowledgments: The authors are grateful to all participants in this study for their support.

Disclosures

None.

\section{References}

1. Schneider M, Bernasch D, Weymann J, Holle R, Bartsch P: Acute mountain sickness: influence of susceptibility, preexposure, and ascent rate. Medicine and science in sports and exercise 2002, 34(12):1886-1891.

2. Beidleman BA, Tighiouart $\mathrm{H}$, Schmid $\mathrm{CH}$, Fulco CS, Muza SR: Predictive models of acute mountain sickness after rapid ascent to various altitudes. Medicine and science in sports and exercise 2013, 45(4):792-800.

3. Bärtsch P, Swenson ER: Clinical practice: Acute high-altitude illnesses. The New England journal of medicine 2013, 368(24):2294-2302.

4. Wilson MH, Newman S, Imray CH: The cerebral effects of ascent to high altitudes. The Lancet Neurology 2009, 8(2):175-191.

5. Imray C, Booth A, Wright A, Bradwell A: Acute altitude illnesses. BMJ (Clinical research ed) 2011, 343:d4943.

6. Roach RC, Hackett PH, Oelz O, Bärtsch P, Luks AM, Maclnnis MJ, Baillie JK: The 2018 Lake Louise Acute Mountain Sickness Score. High altitude medicine \& biology 2018, 19(1):4-6.

7. Meier D, Collet TH, Locatelli I, Cornuz J, Kayser B, Simel DL, Sartori C: Does This Patient Have Acute Mountain Sickness?: The Rational Clinical Examination Systematic Review. Jama 2017, 318(18):1810-1819.

8. Hsu TY, Weng YM, Chiu YH, Li WC, Chen PY, Wang SH, Huang KF, Kao WF, Chiu TF, Chen JC: Rate of ascent and acute mountain sickness at high altitude. Clinical journal of sport medicine : official journal of the Canadian Academy of Sport Medicine 2015, 25(2):95-104.

9. Biondi-Zoccai G, Romagnoli E, Agostoni P, Capodanno D, Castagno D, D'Ascenzo F, Sangiorgi G, Modena MG: Are propensity scores really superior to standard multivariable analysis? Contemporary clinical trials 2011, 32(5):731-740. 
10. Waeber B, Kayser B, Dumont L, Lysakowski C, Tramèr MR, Elia N: Impact of Study Design on Reported Incidences of Acute Mountain Sickness: A Systematic Review. High altitude medicine \& biology 2015, 16(3):204-215.

11. Hackett PH, Rennie D, Levine HD: The incidence, importance, and prophylaxis of acute mountain sickness. Lancet (London, England) 1976, 2(7996):1149-1155.

12. Maggiorini M, Bühler B, Walter M, Oelz O: Prevalence of acute mountain sickness in the Swiss Alps. BMJ (Clinical research ed) 1990, 301(6756):853-855.

13. Chan CW, Lin YC, Chiu YH, Weng YM, Li WC, Lin YJ, Wang SH, Hsu TY, Huang KF, Chiu TF: Incidence and risk factors associated with acute mountain sickness in children trekking on Jade Mountain, Taiwan. Journal of travel medicine 2016, 23(1).

14. Marmura MJ, Hernandez PB: High-altitude headache. Current pain and headache reports 2015, 19(5):483.

15. Hackett PH, Roach RC: High-altitude illness. The New England journal of medicine 2001, 345(2):107114.

16. Sutton JR, Powles AC, Gray GW, Houston CS: Insomnia, sedation, and high altitude cerebral oedema. Lancet (London, England) 1979, 1(8108):165.

17. Wu TY, Ding SQ, Liu JL, Jia JH, Chai ZC, Dai RC, Zhao JZ, Tang QD, Kayser B: Smoking, acute mountain sickness and altitude acclimatisation: a cohort study. Thorax 2012, 67(10):914-919.

18. Vinnikov D, Blanc PD, Steinmaus C: Is Smoking a Predictor for Acute Mountain Sickness? Findings From a Meta-Analysis. Nicotine \& tobacco research : official journal of the Society for Research on Nicotine and Tobacco 2016, 18(6):1509-1516.

19. Luks AM, Swenson ER, Bärtsch P: Acute high-altitude sickness. European respiratory review : an official journal of the European Respiratory Society 2017, 26(143).

20. Beidleman BA, Fulco CS, Glickman EL, Cymerman A, Kenefick RW, Cadarette BS, Andrew SP, Staab JE, Sils IV, Muza SR: Acute Mountain Sickness is Reduced Following 2 Days of Staging During Subsequent Ascent to 4300 m. High altitude medicine \& biology 2018, 19(4):329-338.

21. Schommer K, Wiesegart N, Menold E, Haas U, Lahr K, Buhl H, Bärtsch P, Dehnert C: Training in normobaric hypoxia and its effects on acute mountain sickness after rapid ascent to $4559 \mathrm{~m}$. High altitude medicine \& biology 2010, 11(1):19-25.

22. Fulco CS, Muza SR, Beidleman BA, Demes R, Staab JE, Jones JE, Cymerman A: Effect of repeated normobaric hypoxia exposures during sleep on acute mountain sickness, exercise performance, and sleep during exposure to terrestrial altitude. American journal of physiology Regulatory, integrative and comparative physiology 2011, 300(2):R428-436.

\section{Tables}

Table 1. Incidence of Clinical Outcomes According to the Different High Altitudes in the Fast or Slow Ascent Cohort 
$\begin{array}{llllll}\text { Fast Ascent Cohort } & \text { Slow Ascent Cohort } & \text { P1 } & \text { P2 } & \text { P3 } & \text { P4 }\end{array}$ $\begin{array}{llll}3700 m & 4400 m & 3450 m & 4100 m \\ (n=1283) & (n=757) & (n=346) & (n=314)\end{array}$

\begin{tabular}{lllllllll} 
Old LLS- & 877 & 162 & 105 & 115 & $<0.001$ & $<0.001$ & $<0.001$ & 0.088 \\
AMS & $(68.4 \%)$ & $(21.4 \%)$ & $(30.3 \%)$ & $(36.6 \%)$ & & & & \\
\hline $\begin{array}{l}\text { New LLS- } \\
\text { AMS }\end{array}$ & 720 & 108 & 89 & 101 & $<0.001$ & $<0.001$ & $<0.001$ & 0.068 \\
\hline
\end{tabular}

Clinical

symptoms

\begin{tabular}{|c|c|c|c|c|c|c|c|c|}
\hline Headache & $\begin{array}{l}963 \\
(75.1 \%)\end{array}$ & $\begin{array}{l}171 \\
(22.6 \%)\end{array}$ & $\begin{array}{l}149 \\
(43.1 \%)\end{array}$ & $\begin{array}{l}158 \\
(50.3 \%)\end{array}$ & $<0.001$ & $<0.001$ & $<0.001$ & 0.062 \\
\hline Dizziness & $\begin{array}{l}918 \\
\text { (71.6\%) }\end{array}$ & $\begin{array}{l}167 \\
(22.1 \%)\end{array}$ & $\begin{array}{l}172 \\
(49.7 \%)\end{array}$ & $\begin{array}{l}164 \\
(52.2 \%)\end{array}$ & $<0.001$ & $<0.001$ & $<0.001$ & 0.518 \\
\hline GI upset & $\begin{array}{l}277 \\
(21.6 \%)\end{array}$ & $\begin{array}{l}82 \\
(10.8 \%)\end{array}$ & $\begin{array}{l}48 \\
(13.9 \%)\end{array}$ & $\begin{array}{l}25 \\
(8.0 \%)\end{array}$ & 0.001 & 0.154 & $<0.001$ & 0.016 \\
\hline Insomnia & $\begin{array}{l}835 \\
(65.1 \%)\end{array}$ & $\begin{array}{l}238 \\
(31.4 \%)\end{array}$ & $\begin{array}{l}69 \\
(19.9 \%)\end{array}$ & $\begin{array}{l}73 \\
(23.3 \%)\end{array}$ & $<0.001$ & 0.007 & $<0.001$ & 0.302 \\
\hline Fatigue & $\begin{array}{l}913 \\
(71.2 \%)\end{array}$ & $\begin{array}{l}269 \\
(35.5 \%)\end{array}$ & $\begin{array}{l}136 \\
(39.3 \%)\end{array}$ & $\begin{array}{l}155 \\
(49.4 \%)\end{array}$ & $<0.001$ & $<0.001$ & $<0.001$ & 0.009 \\
\hline Paraesthesia & $\begin{array}{l}595 \\
(46.4 \%)\end{array}$ & $\begin{array}{l}116 \\
(15.3 \%)\end{array}$ & $\begin{array}{l}95 \\
(27.5 \%)\end{array}$ & $\begin{array}{l}92 \\
(29.3 \%)\end{array}$ & $<0.001$ & $<0.001$ & $<0.001$ & 0.600 \\
\hline Constipation & $\begin{array}{l}167 \\
(13.0 \%)\end{array}$ & $\begin{array}{l}72 \\
(9.5 \%)\end{array}$ & $\begin{array}{l}47 \\
(13.6 \%)\end{array}$ & $\begin{array}{l}41 \\
(13.1 \%)\end{array}$ & 0.782 & 0.085 & 0.017 & 0.842 \\
\hline Dyspnea & $\begin{array}{l}438 \\
(34.1 \%)\end{array}$ & $\begin{array}{l}190 \\
(25.1 \%)\end{array}$ & $\begin{array}{l}30 \\
(8.7 \%)\end{array}$ & $\begin{array}{l}63 \\
(20.1 \%)\end{array}$ & $<0.001$ & 0.077 & $<0.001$ & $<0.001$ \\
\hline Cough & $\begin{array}{l}271 \\
(21.1 \%)\end{array}$ & $\begin{array}{l}129 \\
(17.0 \%)\end{array}$ & $\begin{array}{l}27 \\
(7.8 \%)\end{array}$ & $\begin{array}{l}62 \\
(19.8 \%)\end{array}$ & $<0.001$ & 0.293 & 0.025 & $<0.001$ \\
\hline $\begin{array}{l}\text { Chest } \\
\text { distress }\end{array}$ & $\begin{array}{l}521 \\
(40.6 \%)\end{array}$ & $\begin{array}{l}167 \\
(22.1 \%)\end{array}$ & $\begin{array}{l}65 \\
(18.8 \%)\end{array}$ & $\begin{array}{l}66 \\
(21.0 \%)\end{array}$ & $<0.001$ & 0.707 & $<0.001$ & 0.473 \\
\hline Palpitation & $\begin{array}{l}249 \\
(19.4 \%)\end{array}$ & $\begin{array}{l}62 \\
(8.2 \%)\end{array}$ & $\begin{array}{l}30 \\
(8.7 \%)\end{array}$ & $\begin{array}{l}31 \\
(9.9 \%)\end{array}$ & $<0.001$ & 0.373 & $<0.001$ & 0.594 \\
\hline Tinnitus & $\begin{array}{l}323 \\
(25.2 \%)\end{array}$ & $\begin{array}{l}58 \\
(7.7 \%)\end{array}$ & $\begin{array}{l}70 \\
(20.2 \%)\end{array}$ & $\begin{array}{l}50 \\
(15.9 \%)\end{array}$ & 0.056 & $<0.001$ & $<0.001$ & 0.152 \\
\hline Dazzling & $\begin{array}{l}218 \\
(17.0 \%)\end{array}$ & $\begin{array}{l}63 \\
(8.3 \%)\end{array}$ & $\begin{array}{l}30 \\
(8.7 \%)\end{array}$ & $\begin{array}{l}34 \\
(10.8 \%)\end{array}$ & $<0.001$ & 0.193 & $<0.001$ & 0.350 \\
\hline Lethargy & $\begin{array}{l}345 \\
(26.9 \%)\end{array}$ & $\begin{array}{l}99 \\
(13.1 \%)\end{array}$ & $\begin{array}{l}49 \\
(14.2 \%)\end{array}$ & $\begin{array}{l}41 \\
(13.1 \%)\end{array}$ & $<0.001$ & 0.993 & $<0.001$ & 0.680 \\
\hline $\begin{array}{l}\text { Activity } \\
\text { reduction }\end{array}$ & $\begin{array}{l}871 \\
(67.9 \%)\end{array}$ & $\begin{array}{l}321 \\
(42.4 \%)\end{array}$ & $\begin{array}{l}98 \\
(28.3 \%)\end{array}$ & $\begin{array}{l}130 \\
(41.4 \%)\end{array}$ & $<0.001$ & 0.762 & $<0.001$ & $<0.001$ \\
\hline
\end{tabular}


Values are $\mathrm{n}(\%)$. P1 value for comparison between $3700 \mathrm{~m}$ and $3450 \mathrm{~m}$. P2 value for comparison between $4400 \mathrm{~m}$ and $4100 \mathrm{~m}$. P3 value for comparison between $3700 \mathrm{~m}$ and $4400 \mathrm{~m}$. P4 value for comparison between $3460 \mathrm{~m}$ and $4100 \mathrm{~m}$. Gl upset $=$ Gastrointestinal upset .

Table 2a. Risk Factors of Old LLS-AMS and New LLS-AMS at Different High Altitudes in the Fast Ascent Cohort after Univariate Analysis

\begin{tabular}{|c|c|c|c|c|}
\hline & \multicolumn{2}{|l|}{$3700 \mathrm{~m}$} & \multicolumn{2}{|l|}{$4400 m$} \\
\hline & Old LLS-AMS & New LLS-AMS & Old LLS-AMS & New LLS-AMS \\
\hline Age, years & $1.02(0.99,1.05)$ & $1.01(0.99,1.04)$ & $1.05(1.00,1.10)^{\star}$ & $1.02(0.97,1.08)$ \\
\hline Height, cm & $0.99(0.97,1.02)$ & $0.99(0.96,1.01)$ & $0.98(0.95,1.02)$ & $1.00(0.96,1.05)$ \\
\hline Weight, kg & $1.00(0.99,1.02)$ & $1.00(0.99,1.02)$ & $1.01(0.99,1.03)$ & $1.02(0.99,1.05)$ \\
\hline BMI, kg.m-2 & $1.03(0.97,1.09)$ & $1.02(0.97,1.07)$ & $1.06(0.98,1.15)$ & $1.09(1.00,1.20)$ \\
\hline \multicolumn{5}{|l|}{ Smoking, n(\%) } \\
\hline non & 1 & 1 & 1 & 1 \\
\hline previous & $0.84(0.58,1.21)$ & $1.07(0.76,1.51)$ & $1.05(0.60,1.81)$ & $0.85(0.45,1.64)$ \\
\hline current & $0.74(0.56,0.97)^{\star}$ & $0.85(0.66,1.10)$ & $1.18(0.78,1.79)$ & $1.01(0.63,1.63)$ \\
\hline \multicolumn{5}{|l|}{ Nationality, n(\%) } \\
\hline Han & 1 & 1 & 1 & 1 \\
\hline non-Han & $0.72(0.50,1.04)$ & $0.79(0.55,1.13)$ & $0.51(0.27,0.96)^{\star}$ & $0.60(0.29,1.24)$ \\
\hline \multicolumn{5}{|l|}{ Education, n(\%) } \\
\hline university & 1 & 1 & 1 & 1 \\
\hline high school & $1.09(0.79,1.50)$ & $1.08(0.80,1.46)$ & $1.15(0.70,1.88)$ & $1.15(0.64,2.05)$ \\
\hline under high school & $0.80(0.57,1.13)$ & $0.77(0.56,1.07)$ & $0.94(0.55,1.62)$ & $0.98(0.52,1.87)$ \\
\hline unknown & $0.71(0.36,1.37)$ & $0.62(0.32,1.17)$ & $1.40(0.46,4.24)$ & $1.74(0.52,5.87)$ \\
\hline \multicolumn{5}{|c|}{ History of HA exposure within 1 year, $n(\%)$} \\
\hline no & 1 & 1 & 1 & 1 \\
\hline yes & $0.99(0.72,1.35)$ & $0.93(0.69,1.24)$ & $0.94(0.60,1.49)$ & $0.79(0.45,1.36)$ \\
\hline unknown & $0.95(0.73,1.24)$ & $0.88(0.68,1.13)$ & $1.34(0.90,2.01)$ & $1.14(0.71,1.82)$ \\
\hline
\end{tabular}

Values are $\beta / O R(95 \% \mathrm{Cl})$; * means $p$-value $<0.05$, and $* *$ means $p$-value $<0.01$. Other abbreviations as in Table 1 and Table 2. 
Table 2b. Risk Factors of Old LLS-AMS and New LLS-AMS at Different High Altitudes in the Slow Ascent Cohort after Univariate Analysis

\begin{tabular}{|c|c|c|c|c|}
\hline & $3450 \mathrm{~m}$ & & $4100 \mathrm{~m}$ & \\
\hline & Old LLS-AMS & New LLS-AMS & Old LLS-AMS & New LLS-AMS \\
\hline Age, years & $1.02(0.95,1.10)$ & $1.00(0.92,1.08)$ & $0.92(0.84,1.00)$ & $0.92(0.85,1.01)$ \\
\hline Height, cm & $0.98(0.93,1.03)$ & $1.00(0.95,1.05)$ & $0.97(0.92,1.02)$ & $0.97(0.92,1.02)$ \\
\hline Weight, kg & $0.98(0.94,1.01)$ & $0.98(0.95,1.02)$ & $0.98(0.94,1.01)$ & $0.97(0.94,1.01)$ \\
\hline $\mathrm{BMI}, \mathrm{kg} \cdot \mathrm{m}^{-2}$ & $0.93(0.82,1.05)$ & $0.93(0.82,1.06)$ & $0.95(0.84,1.08)$ & $0.94(0.83,1.07)$ \\
\hline \multicolumn{5}{|l|}{ Smoking, n(\%) } \\
\hline non & 1 & 1 & 1 & 1 \\
\hline previous & $1.04(0.55,1.99)$ & $1.15(0.58,2.27)$ & $1.00(0.52,1.95)$ & $1.42(0.71,2.84)$ \\
\hline current & $1.04(0.61,1.77)$ & $1.13(0.64,1.99)$ & $1.48(0.86,2.53)$ & $1.95(1.10,3.45)$ * \\
\hline \multicolumn{5}{|l|}{ Nationality, n(\%) } \\
\hline Han & 1 & 1 & 1 & 1 \\
\hline non-Han & $0.39(0.17,0.91)$ * & $0.51(0.22,1.18)$ & $0.50(0.23,1.05)$ & $0.38(0.16,0.89) *$ \\
\hline \multicolumn{5}{|l|}{ Education, n(\%) } \\
\hline university & 1 & 1 & 1 & 1 \\
\hline high school & $1.00(0.52,1.94)$ & $1.07(0.53,2.17)$ & $1.22(0.63,2.36)$ & $1.45(0.72,2.92)$ \\
\hline under high school & $0.84(0.38,1.85)$ & $0.89(0.38,2.06)$ & $1.06(0.49,2.28)$ & $1.55(0.69,3.47)$ \\
\hline unknown & $1.20(0.42,3.40)$ & $1.31(0.44,3.89)$ & $0.71(0.20,2.55)$ & $0.71(0.17,2.93)$ \\
\hline no & 1 & 1 & 1 & 1 \\
\hline yes & $1.32(0.65,2.67)$ & $1.12(0.53,2.39)$ & $1.26(0.55,2.85)$ & $0.89(0.37,2.14)$ \\
\hline unknown & $0.63(0.29,1.39)$ & $0.57(0.24,1.34)$ & $0.80(0.39,1.61)$ & $0.74(0.35,1.55)$ \\
\hline
\end{tabular}

Values are $\beta / O R(95 \% \mathrm{Cl})$; * means $p$-value $<0.05$, and $* *$ means $p$-value $<0.01$. Other abbreviations as in Table 1 and Table 2.

Table 3. Main Clinical Outcomes in the Overall Propensity Score-Matched Cohort According to Different Plateau Ascent Protocols 


\begin{tabular}{lllll}
\hline & Univariate & Multivariate & Univariate & Multivariate \\
\hline Old LLS-AMS & $4.2(2.9,5.9) * *$ & $4.3(3.0,6.1) * *$ & $0.4(0.3,0.6) * *$ & $0.4(0.2,0.5) * *$ \\
\hline New LLS-AMS & $3.0(2.1,4.3) * *$ & $3.0(2.1,4.4) * *$ & $0.3(0.2,0.5) * *$ & $0.3(0.2,0.5) * *$ \\
\hline Headache & $3.4(2.4,4.9) * *$ & $3.5(2.4,5.1) * *$ & $0.3(0.2,0.4) * *$ & $0.3(0.2,0.4) * *$ \\
\hline Dizziness & $2.3(1.6,3.2) * *$ & $2.3(1.6,3.2) * *$ & $0.2(0.2,0.4) * *$ & $0.2(0.2,0.3) * *$ \\
\hline GI upset & $1.6(1.0,2.5)$ & $1.6(1.0,2.5)$ & $0.9(0.5,1.7)$ & $0.9(0.5,1.6)$ \\
\hline Fatigue & $3.1(2.2,4.4) * *$ & $3.2(2.2,4.5) * *$ & $0.5(0.4,0.8) * *$ & $0.5(0.4,0.7) * *$ \\
\hline Insomnia & $6.4(4.4,9.3) * *$ & $6.5(4.4,9.5) * *$ & $1.6(1.1,2.3) *$ & $1.6(1.1,2.4) *$
\end{tabular}

Values are $\beta / O R(95 \% \mathrm{Cl}) p$ Value; * means $p$-value $<0.05$, and $* *$ means $p$-value $<0.01$; Other abbreviations as in Table 1 and Table 2. Mulitivariate analysis were adjusted for age, BMI, smoking, nationality, education and plateau history.

Figures 


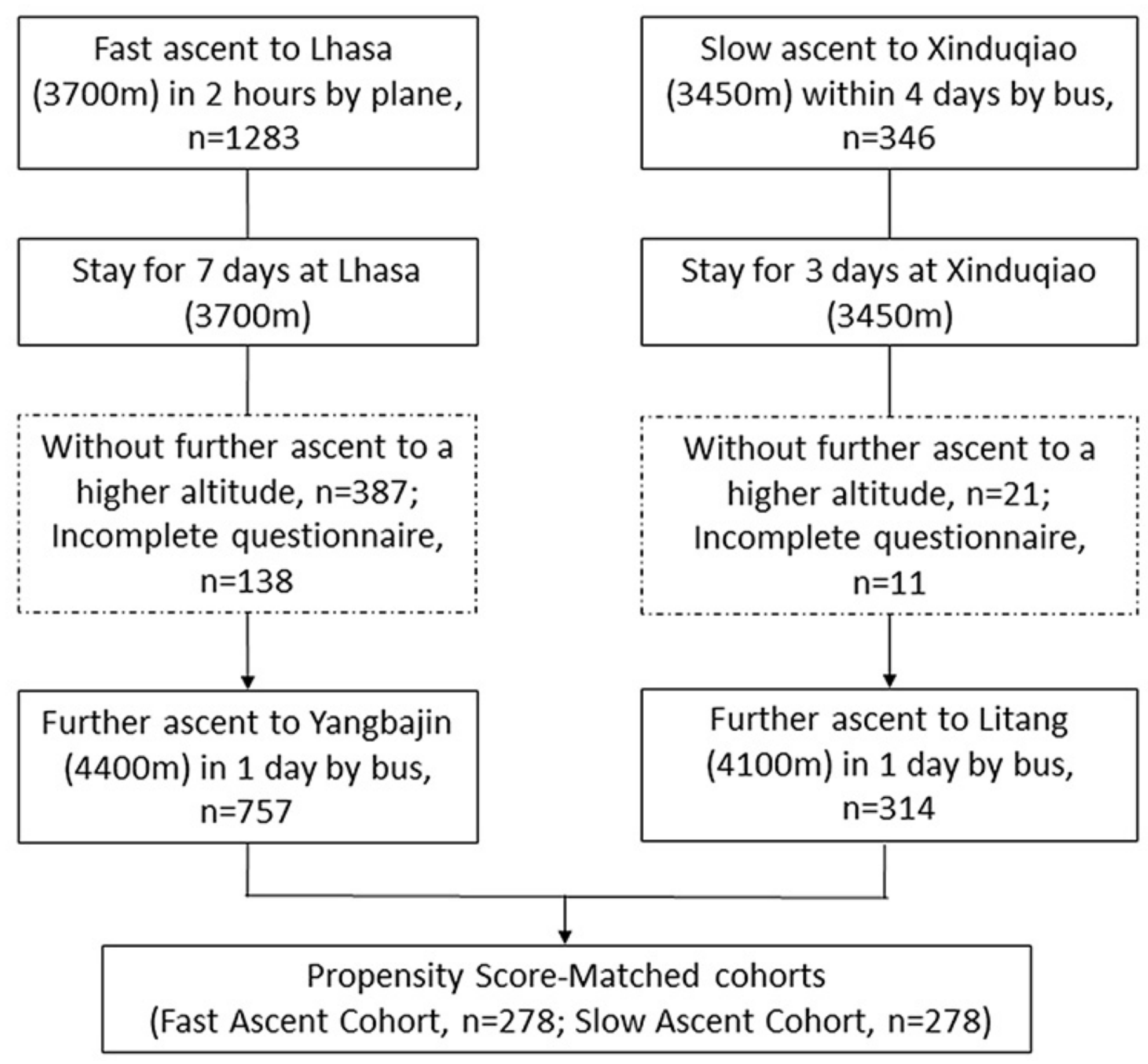

Figure 1

Study flow chart. 

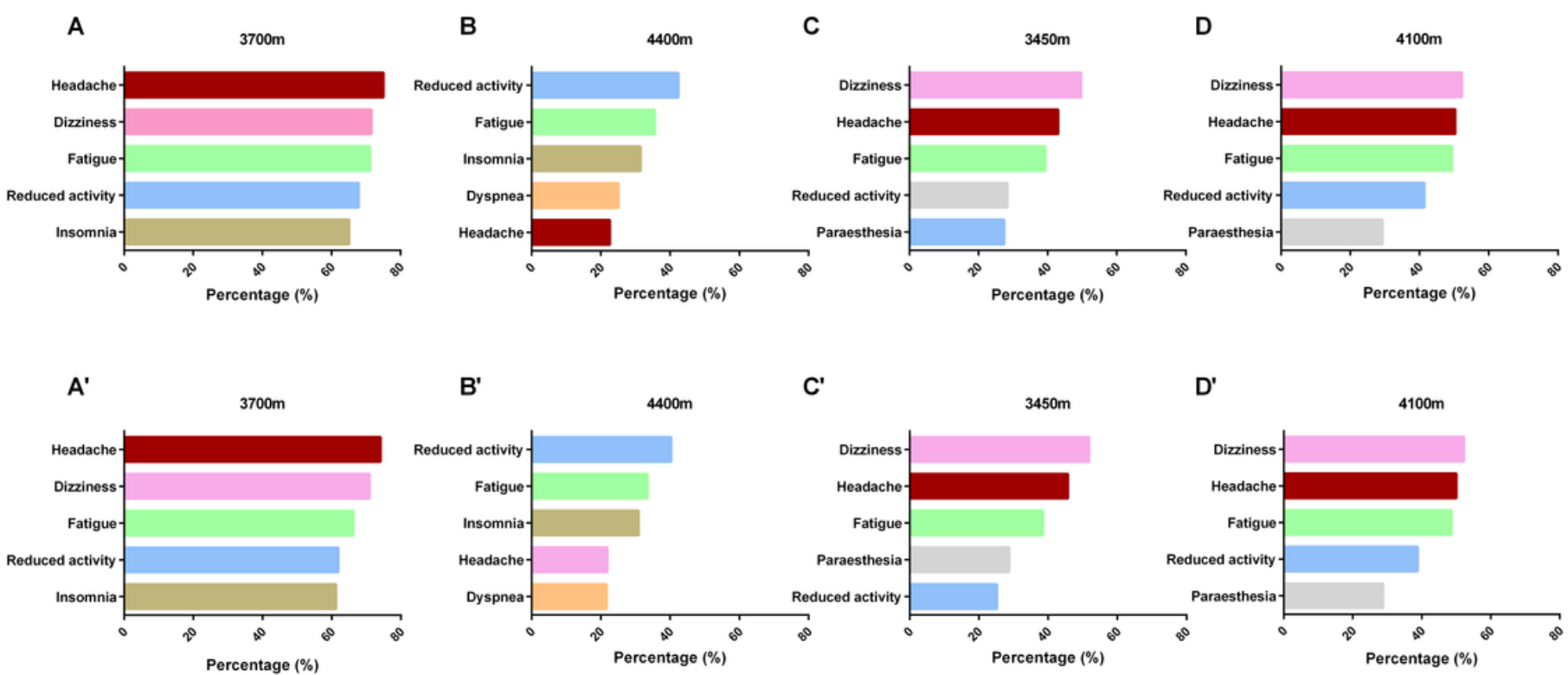

Figure 2

Evolution of the Clinical Symptoms According to the Different High Altitudes in the Entire and Propensity Score-Matched Cohorts. Figure (A-D) showed the ranking five clinical symptoms in the entire cohorts and Figure $\left(A^{\prime}-D^{\prime}\right)$ showed the ranking five clinical symptoms in the propensity score-matched cohort.

\section{Supplementary Files}

This is a list of supplementary files associated with this preprint. Click to download.

- Supplementaltables.doc 